\title{
Screening diagnostic biomarkers of OSCC via an LCM-based proteomic approach
}

\author{
RUINAN WANG ${ }^{1,2^{*}}$, YAO YUAN ${ }^{1 *}$, YUQIAO ZHOU $^{1 *}$, DUNFANG ZHANG $^{1}$, LEWEI ZHANG $^{3}$, XIN ZENG $^{1}$, \\ NING JI ${ }^{1}$, MIN ZHOU ${ }^{1}$, XINHUA LIANG ${ }^{1}$, YU CHEN ${ }^{1}$, NING GENG ${ }^{1}$, JING LI ${ }^{1}$ and QIANMING CHEN ${ }^{1}$ \\ ${ }^{1}$ State Key Laboratory of Oral Diseases, National Clinical Research Center for Oral Diseases, West China Hospital \\ of Stomatology; ${ }^{2}$ School of Life Science and Key Laboratory of Bio-Resources and Eco-Environment, Ministry of \\ Education, Sichuan University, Chengdu, Sichuan 610041, P.R. China; ${ }^{3}$ British Columbia Cancer Agency/Cancer \\ Research Center and Faculty of Dentistry, University of British Columbia, Vancouver, BC V6T 1Z4, Canada
}

Received January 11, 2018; Accepted June 7, 2018

DOI: 10.3892/or.2018.6610

\begin{abstract}
The current standard for the diagnosis of oral squamous cell carcinoma (OSCC) is based on the histologic examination of hematoxylin and eosin-stained sections; however, the discrimination among normal tissue, pre-cancerous lesions and cancerous lesions can be difficult. The aim of the present study was to identify proteins with diagnostic significance in differentiating or predicting oral mucosal carcinogenesis. Proteomic profiling based on the laser capture microdissection of formalin-fixed, paraffin-embedded samples was performed, followed by liquid chromatography-tandem mass spectrometry (LC/MS) analysis. Immunohistochemistry (IHC) was used to evaluate the results. IHC of cytokeratins (CKs) was performed in neck dissection treatment cases. The accuracy rate and $95 \%$ confidence intervals (CIs) were used to evaluate the value of CKs as biomarkers of OSCC. A lymph node metastasis mouse model was used to validate the selected biomarkers. Among the proteins identified using LC/MS, several CKs exhibited significant differential expression patterns between the cancerous and para-cancerous tissues. The IHC results showed that negative staining of CK4 and CK10/13 distinguished cancerous from para-cancerous tissues with an accuracy of 90\% (95\% CI, 0.68-0.99) and 75\% (95\% CI, 0.51-0.91), respectively. Furthermore, the positive staining of CK14 and CK17
\end{abstract}

Correspondence to: Dr Ning Geng or Dr Jing Li, State Key Laboratory of Oral Diseases, National Clinical Research Center for Oral Diseases, West China Hospital of Stomatology, Sichuan University, 14 Renmin South Road Section 3, Chengdu, Sichuan 610041, P.R. China

E-mail: gengning@scu.edu.cn

E-mail: lijing1984@scu.edu.cn

${ }^{*}$ Contributed equally

Key words: surgical resection, biomarker, proteomics, metastasis, prognosis, cytokeratin clearly distinguished cancerous from para-cancerous lesions with an accuracy of $100 \%$ (95\% CI, 83-100\%) and 90\% (95\% CI, 0.68-0.99), respectively. There was also CK14-positive staining in micro-metastases of lymph nodes in the clinical samples and in an animal model.

\section{Introduction}

Oral cancer is a serious and increasingly prevalent disease in several regions worldwide. Oral and pharyngeal cancer, grouped together, constitute the sixth most common type of cancer globally. The estimated annual incidence is $\sim 275,000$ for oral cancer, and two thirds of these cases occur in developing countries (1). In China, $\sim 8 / 100,000$ of the population experience oral cancer per year according to the World Health Organization (2). Of these tumors, $>90 \%$ are oral squamous cell carcinoma (OSCC). Despite advances in diagnosis and treatment, the 5-year survival rate of patients with OSCC is no more than $50 \%$ (3). In the late stages of tongue cancer, the 5-year survival rate has markedly decreased to 5\% [National Cancer Institute's Surveillance, Epidemiology, and End Results program (2003-2009)] (https://seer.cancer.gov/archive/csr).

The histologic examination of routine hematoxylin and eosin (H\&E)-stained sections is the current standard used in the diagnosis of OSCC; however, the discrimination of normal tissue, pre-cancerous lesions, cancerous lesions, and micrometastasis in lymph nodes based on H\&E-stained sections of the oral epithelium can be difficult $(4,5)$. It is well established that the development of oral cancer consists of two steps, the initial presence of a precursor lesion and its subsequent development into cancer (6). Although certain morphological changes may underlie the process of pre-cancerous lesions developing into cancer and thus provide certain clues for assessing the risk of malignancy of lesions, pathological decisions based on such observations can be subjective and even controversial. For example, opinions vary among pathologists regarding the interpretation of the extent of dysplasia in the same lesion. Therefore, the identification of relatively objective biomarkers that can be used to identify oral malignancy or micrometastasis in lymph nodes will benefit the evaluation and diagnosis of patients with OSCC (7). 
To identify proteins capable of differentiating OSCC tissues from para-cancerous tissues in the same patient, the present study performed assays directly on diseased tissues using stringent protocols and a well-designed workflow. First, a vast archive of biopsied and excised specimens, which had been processed for histological diagnosis, formalin-fixed, and paraffin-embedded (FFPE), were accessed. These specimens represent a unique source of morphologically defined and disease-specific samples, containing substantial protein information and the accompanying patient clinical information, and have already been applied in the assessment of novel and classical biomarkers (8-10).

Inevitably, investigations based on FFPE tissues must account for the loss of protein in FFPE samples, as the process of fixation and long duration of storage may induce degradation and alteration of proteins in FFPE tissues. Therefore, the present study used a commercially available protein extraction kit, which enabled the extraction of as many protein/peptides as possible from relatively newly-preserved FFPE tissues (within 6 months). Mass spectrometry technologies have been widely used for biomarker screening in human diseases (11). Another important technical aspect in the biomarker identification process in the present study was the use of laser capture microdissection (LCM), which allows the identification and capture of targeted cells from complex clinical specimens without interference from the tumor stroma (12). Using LCM, oral cancer cells, pre-cancerous cells, and normal mucosal epithelial cells were obtained from FFPE specimens derived from the same individual, on which proteomic analysis using liquid chromatography-tandem mass spectrometry (LC/MS) was performed. This facilitated the high-throughput proteomic analysis of differentially expressed proteins (10).

The approach used in the present study to quantify proteome changes in oral cancer tissues allowed for the highthroughput and unbiased identification of novel proteomic changes in OSCC. This LCM-FFPE-proteomic and LC/MS analysis approach, followed by IHC validation in formalinfixed tissues, serves as a reliable platform for the identification of diagnostic biomarkers for OSCC.

\section{Materials and methods}

Clinical samples and histological analysis. Archived FFPE tissue samples, which had been pathologically diagnosed within the prior 6 months, were classified into two groups: Group 1 consisted of three paired tissue samples. Each paired sample was derived from the same individual and consisted of OSCC lesions and their corresponding adjacent normal mucosal lesions. H\&E staining was used to determine the tumor tissue boundaries. The distance from the normal tissues to cancerous tissues was $\sim 1-2 \mathrm{~mm}$.

Group 2 also consisted of three paired cases (leukoplakia carcinogenesis tissue), and each was derived from the same individual, including paired normal oral mucosa, oral leukoplakia with mild-moderate dysplasia, and OSCC lesions. H\&E staining was used to determine the boundaries of the tumor tissues or leukoplakia with mild-moderate dysplastic tissues. The distance from the normal tissues to leukoplakia with mild-moderate dysplasia tissues, or leukoplakia with mild-moderate dysplasia tissues to cancerous tissues was also
1-2 mm. For cytokeratin (CK) immunohistochemistry (IHC) analysis and validation, three groups of samples were used: Group 1: 20 FFPE specimens diagnosed as OSCC within the most recent 5 years, which included normal, pre-cancerous, and cancerous areas; Group 2: 20 neck dissection specimens with lymph node micro-metastases; and Group 3:10 samples diagnosed with non-metastatic lymph nodes.

The histological analysis and diagnosis were performed by a pathologist (Dr Ning Geng) and then confirmed by a second pathologist (Dr Yu Chen) of the State Key Laboratory of Oral Diseases, National Clinical Research Center for Oral Diseases, West China Hospital of Stomatology (Sichuan, China). All tissue samples were obtained from the Oral Pathology Archives of West China Hospital of Stomatology, and were fixed and paraffin-embedded using standard procedures. The Institutional Review Board of the West China College of Stomatology, Sichuan University approved the protocol.

LCM using FFPE sections. The FFPE oral cancer tissue sections (5- $\mu \mathrm{M}$ thick) on Arcturus ${ }^{\circledR}$ PEN membrane glass slides (Thermo Fisher Scientific, Inc., Waltham, MA, USA) were deparaffinized in xylene, hydrated and stained in hematoxylin, followed by dehydration. For LCM, the stained uncovered Arcturus ${ }^{\circledR}$ PEN membrane glass slides were air dried and $\sim 10,000$ cells were captured onto CapSure ${ }^{\circledR}$ HS LCM caps (Thermo Fisher Scientific, Inc.) using an ArcturusXT ${ }^{\text {тм }}$ microdissection system (Thermo Fisher Scientific, Inc.). The caps were transferred to a $0.5-\mathrm{ml}$ sterile Eppendorf tube for protein extraction.

Protein extraction from captured cells derived from FFPE tissues. The protein lysates were prepared according to the manufacturer's protocol (Liquid Tissue kit; Expression Pathology, Inc., Gaithersburg, MD, USA). The procured cells ( $\sim 10,000$ cells for each set of tissues) were suspended in $20 \mu \mathrm{l}$ of proprietary Liquid Tissue buffer (Expression Pathology, Inc.) and heated at $95^{\circ} \mathrm{C}$ for $90 \mathrm{~min}$. Following cooling for 2 min on ice, $1 \mu 1$ of trypsin reagent was added and incubated at $37^{\circ} \mathrm{C}$ for $1 \mathrm{~h}$ with vigorous shaking for $1 \mathrm{~min}$ at $20 \mathrm{~min}$ intervals. The samples were then incubated at $37^{\circ} \mathrm{C}$ for $18 \mathrm{~h}$ followed by heating at $95^{\circ} \mathrm{C}$ for $5 \mathrm{~min}$, and then stored at $-20^{\circ} \mathrm{C}$ prior to use for LC/MS analysis.

Proteomic analysis by LC/MS. The FFPE-extracted samples were processed, quantified, and analyzed in a Thermo LTQ 7000 high-performance liquid chromatography (HPLC)-mass spectrophotometer (LTQ; Thermo Fisher Scientific, Inc.). Protein separation was performed on a Thermo Scientific Surveyor HPLC system with Hypersil GOLD columns (Thermo Fisher Scientific, Inc.). The spectra were searched against the NCBI Protein Bank human protein database (https://www.ncbi.nlm.nih.gov/protein/) from the European Bioinformatics Institute using SEQUEST 3.2 (Thermo Fisher Scientific, Inc.). The results were further filtered using software developed in-house to determine unique peptides and proteins, which has a predicted error of $<1.5 \%$.

IHC analysis. Archived FFPE tissue blocks from the Department of Oral Pathology, West China College of Stomatology, Sichuan University were used for histopathologic 

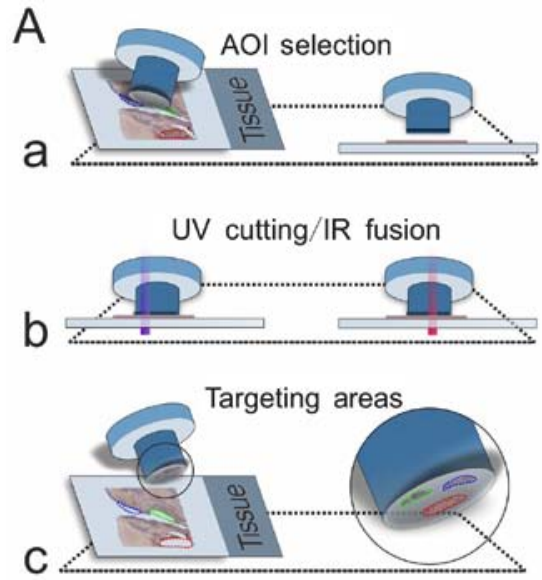

Protein extraction
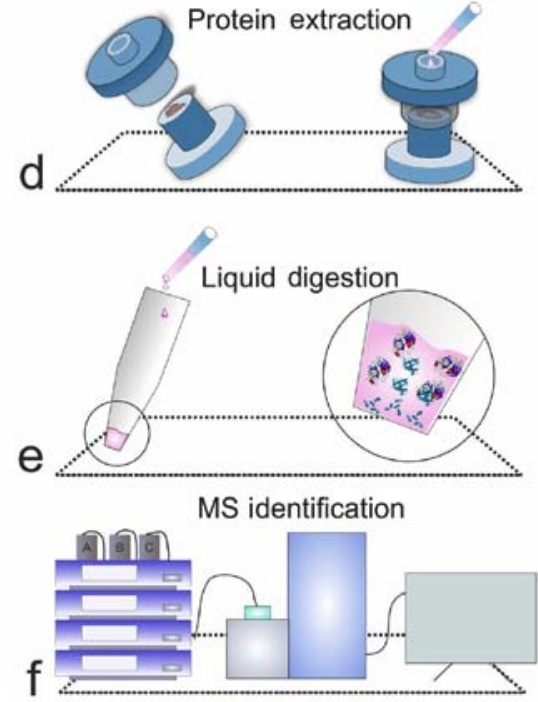

B
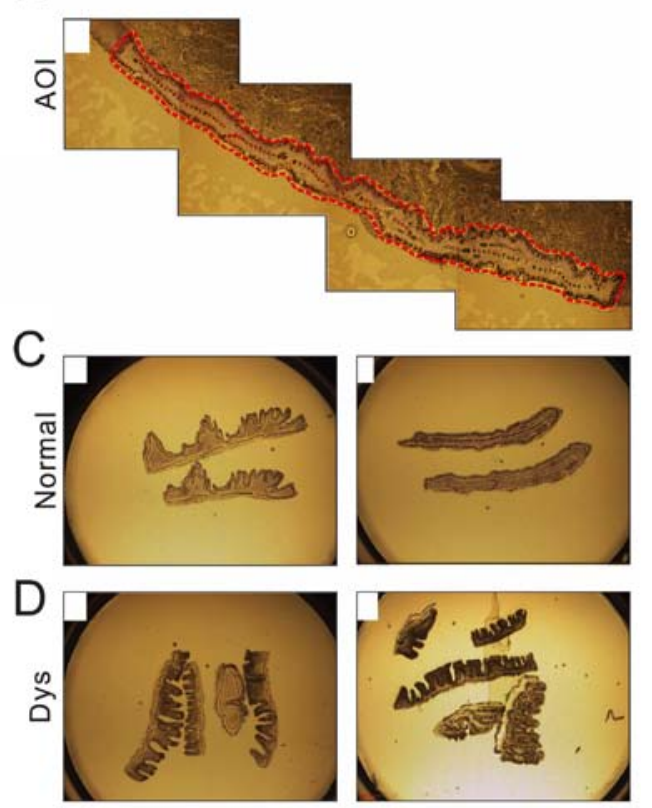

$\mathrm{E}$

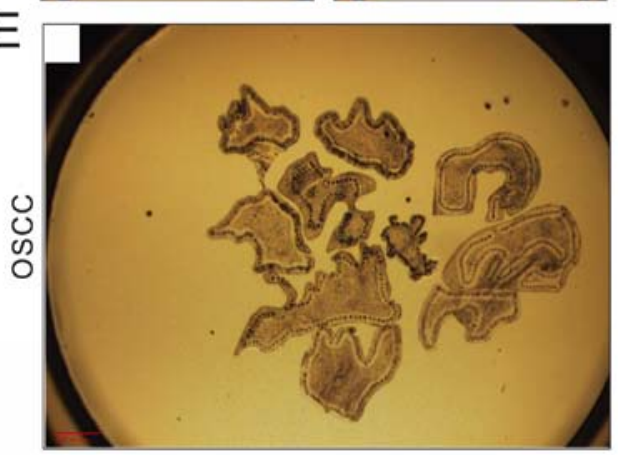

Figure 1. Proteomic profiling based on LCM and LC/MS analyses of clinical FFPE tissues. (A) Schematic illustrating the workflow of LCM-LC/MS analyses of clinical FFPE tissues. The steps are as follows: (a) AOI selected according to the staining; (b) AOI cut by UV and followed by IR fusion; (c) targeted AOI separated by the cup; (d) protein extraction; (e) proteins digested via liquid enzyme digestion; (f) digested peptides identified via LC-MS/MS. Representative images (x4) of the microdissection of the oral mucosal epithelium by LCM in FFPE sections showing (B) AOI, (C) normal, (D) Dys and (E) OSCC tissues. FFPE, formalin-fixed, and paraffin-embedded; LCM, laser capture microdissection; LC/MS, liquid chromatography-tandem mass spectrometry; AOI, area of interest; Dys, dysplasia; OSCC, oral squamous cell carcinoma.

and immunohistochemical analysis. EnVision/HRP, a two-step IHC staining technique, was used for IHC staining (EnVision Detection system, Peroxidase/DAB, rabbit/mouse; code no. 500750; Gene Tech Co., Ltd., Shanghai, China). Sections (4- $\mu \mathrm{M})$ were prepared from the FFPE blocks on adhesive glass slides. Antigen retrieval was achieved by boiling the sections in $0.01 \mathrm{M}$ citrate buffer ( $\mathrm{pH}$ 6.0) in a high-pressure cooker for $3 \mathrm{~min}$. Primary antibodies used in this procedure were as follows: CK4 (rabbit monoclonal; 1:200; cat. no. M07410; Boster Biological Technology, Pleasanton CA, USA); CK14 (rabbit monoclonal; 1:200; cat. no. ZA-0540; ZSGB-BIO, Beijing, China); CK10/13 (mouse monoclonal; 1:30; cat. no. ZM-0314; ZSGB-BIO); CK17 (rabbit monoclonal; 1:200; cat. no. ZA-0551; ZSGB-BIO); and Pan CK (mouse monoclonal; 1:200; cat. no. ZM-0069; ZSGB-BIO). Duplicate sections were incubated overnight in $4^{\circ} \mathrm{C}$ with primary antibody.

Lymph node metastasis mouse model. Female BALB/c-nu/nu nude mice (5-6 weeks old) weighing 18-20 g $(n=6)$ were used in the present study. Six mice were housed in a specific pathogen free class laboratory animal room, which was maintained at $22 \pm 2^{\circ} \mathrm{C}$ on a regular light-dark cycle. All animals were provided with food and water ad libitum. The use of animals in the experiments was approved by the State Key Laboratory of Oral Diseases Research Ethics Committee.

HSC-3 cells were purchased from the Cell Bank of Japanese Collection of Research Bioresource (JCRB; Shinjuku, Japan). This cell was cultured in DMEM (Sigma-Aldrich; Merck $\mathrm{KGaA}$ ) with $10 \%$ fetal bovine serum (FBS) in a humidified atmosphere with $5 \% \mathrm{CO}_{2}$. The cells were collected separately in the logarithmic phase, washed twice in 1X PBS, and added to fresh medium without serum, cells were counted and the cell concentration was adjusted to $2 \times 10^{7}$ cells $/ \mathrm{ml}$. On the inside of the oral buccal mucosa of the nude mice, the cells were inoculated in a $50-\mu 1$ cell suspension ( $1 \times 10^{6}$ cells/ mouse). The mice were sacrificed at 45 days and the neck lymph nodes were collected, for the production of paraffinized sections for H\&E staining and immunohistochemical staining.

Statistical analysis. The statistical analysis was conducted using SPSS version 19 (IBM Corp., Armonk, NY, USA). The rate of accuracy and $95 \%$ confidence intervals (CIs) were used 
Table I. Representative proteins identified using liquid chromatography-tandem mass spectrometry analysis.

Classification

Representative proteins

\section{Cell structure \\ Cytoskeletal proteins \\ Desmosomal proteins \\ Membrane-related proteins \\ Cell differentiation}

Cell metabolism

Cell-cycle regulation

Cell damage and repair

Genetic regulation

Signaling pathway

Transportation
CK16, CK6, CK14, CK17, CK1, Actin, Tubulin, Plectin-1

Desmoplakin, Plakophilin, Desmoglein

Annexin I, Annexin A2

Involucrin, Small proline-rich protein $1 \mathrm{~B}$, Small proline-rich protein $1 \mathrm{~A}$, Small proline-rich protein 3, Cornifin A, Cornifin B

Pyruvate kinase 3, Glyceraldehyde-3-phosphate dehydrogenase, ATP synthase, Enolase, Tyr3/Trp5-mono-oxygenase activation protein, Calgranulin B, Calgranulin A.

Eukaryotic translation elongation factor 1

Heat shock protein 27, Heat shock protein 70, Heat shock protein 90.

H4 histone family, MYC promoter-binding protein

Calmodulin-like 3, S100 Calcium-binding protein A8, S100 Calcium-binding protein A9

Serum albumin precursor

CK, cytokeratin.

to evaluate the effects of $\mathrm{CKs}$ in differentiating OSCC from para-cancerous tissues, and the $95 \% \mathrm{CI}$ of was performed by using Bootstrap test.

\section{Results}

Proteomic profiling based on LCM of FFPE tissues represents a reliable approach for biomarker identification. In the present study, the Arcturus LCM platform was used, which features three functions: Cut, capture, and the combination of the two. During the procedure, the platform was used together with CapSure ${ }^{\circledR}$ HS LCM caps. An elevated ring surrounds the cap, and the captured cells adhere to this. Proteins extracted from these FFPE samples are amenable to LC/MS analysis. The detailed experimental procedures are shown in Fig. 1A. For the FFPE samples, 10,000-20,000 (two caps) cells were obtained from each type of tissue, although in one case of mucosal epithelial cells, 6,000-10,000 cells were obtained. The prepared OSCC tissues/cells micro-dissected for further analysis using LCM are shown in Fig. 1B-E.

Detection of differentially expressed proteins between OSCC tissues and tumor-adjacent normal tissues. To obtain insights into the proteins distinctively expressed in OSCC tissues and adjacent normal tissues, paired OSCC and para-cancerous normal mucosal tissues were analyzed. Each paired sample was derived from the same individual, and the group consisted of three paired tissue samples (Fig. 2A). The LC/MS proteomics platform used in this analysis enabled the identification of 143 total proteins in OSCC tumor tissues and 133 adjacent normal tissues, representing a diverse array of molecular functions, including cytoskeleton proteins; a membrane protein; desmosomal proteins; and proteins involved in cell differentiation, cell metabolism, cell-cycle regulation, cell damage and repair, genetic regulation, a signaling pathway, and transportation (Table I). Among these, 82 proteins were shared across normal and OSCC tissues (Fig. 2B), whereas tumor-adjacent tissues exhibited high levels of CK4, 13 and 10.

The proteins detected in the normal oral squamous epithelium included CK12 and cornulin, also known as tumorrelated protein, which is a cytoplasmic protein considered to be involved in epidermal differentiation and the epithelial immune response. Several studies have shown that its downregulation is linked to tumor progression $(13,14)$. The proteins expressed uniquely in normal tissues indicated that their biological functions may include maintaining normal epithelial differentiation and suppressing tumors.

Proteins differentially expressed in normal, pre-cancerous, and cancerous oral mucosal epithelial tissues may be effective biomarkers for OSCC diagnosis. To obtain insights into the proteins differentially expressed at different stages of oral carcinogenesis, including normal epithelium, pre-cancerous tissues (OLK), and OSCC tissues, paired normal oral mucosa and oral leukoplakia with mild-moderate dysplasia were included in the analysis. A total of 53 proteins were expressed in all three tissue sets, including CKs and desmosomal proteins (Fig. 2C; Table I). Notably, CK4 was the most abundant in normal tissues, and its expression level was significantly lower in the OLK and OSCC tissues. By contrast, the protein expression levels of CK6, 14, 16 and 17; desmoplakin; actin $\gamma 1$; eukaryotic translation elongation factor 1; and $\mathrm{H} 4$ histone were found to be higher in the tumor samples than in the OLK samples, and their levels continually decreased from the OLK samples to the normal samples. This shift in the distribution pattern may be indicative of the importance of these proteins in oral carcinogenesis.

A total of 13 proteins were found to be expressed at high levels specifically in OSCC and OLK tissues. The majority of CKs, including CK3, 5, 6, 14 and 16, had higher expression scores in OSCC tissues than in adjacent OLK tissues or normal tissues. Notably, the expression of CK17 was 10 -fold 
A
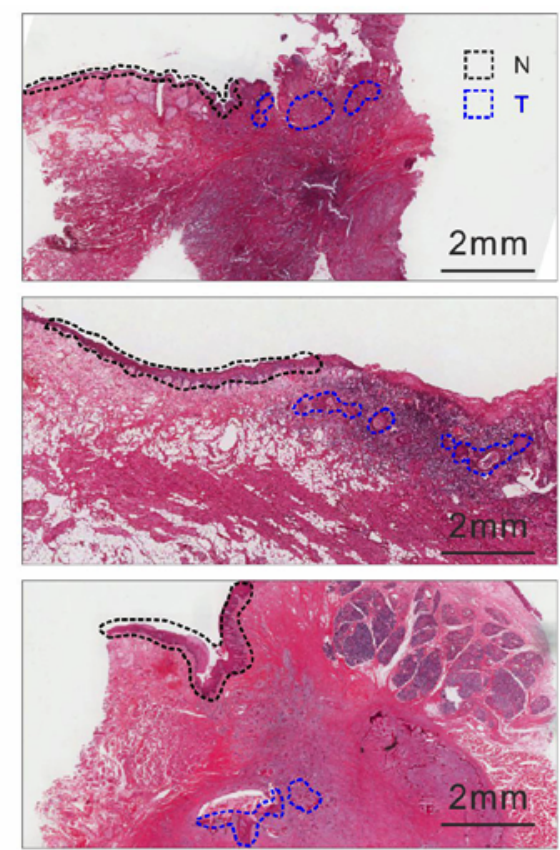
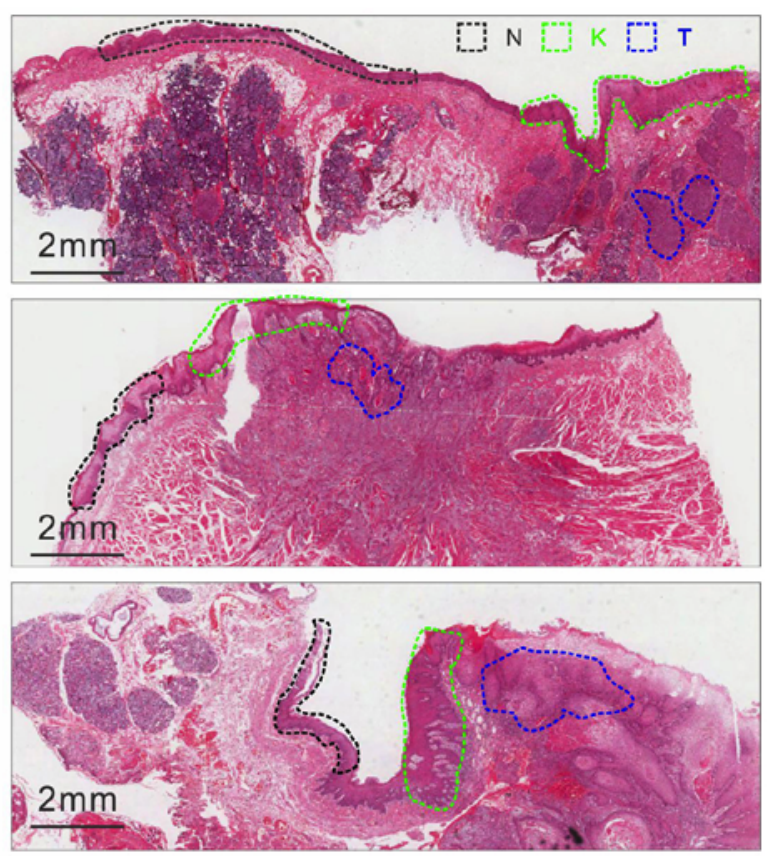

B

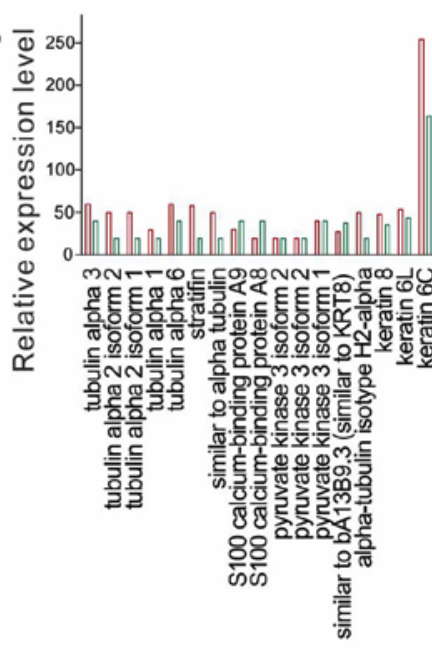

$\mathrm{C}$

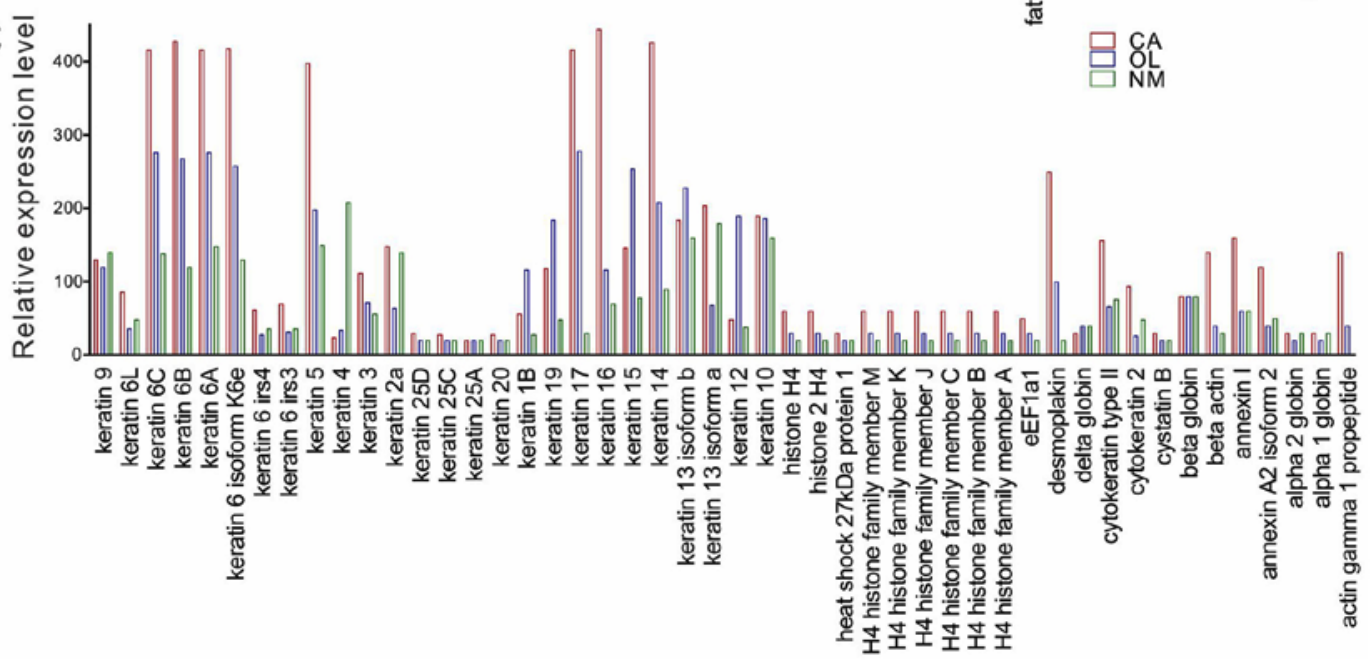

Figure 2. Panoramic view of H\&E staining and protein expression in clinical samples analyzed with LC/MS. (A) Representative H\&E staining images of adjacent N, T, and/or K regions in six cases used for LC/MS analysis. Group 1 cases are on the left; Group 2 cases are on the right. Scale bar, $2 \mathrm{~mm}$. (B) LC/MS analysis of proteins shared by OSCC tissues and adjacent normal surrounding mucosa tissues. y-axis represents the relative level of the proteins. (C) LC/MS analysis of proteins shared by OSCC (CA), pre-cancerous lesions (OLK) and normal (NM) tissues. y-axis represents the relative level of the proteins. H\&E, hematoxylin and eosin; LC/MS, liquid chromatography-tandem mass spectrometry; OSCC, oral squamous cell carcinoma; N, normal; T, tumor; K, pre-cancerous lesions. 
A

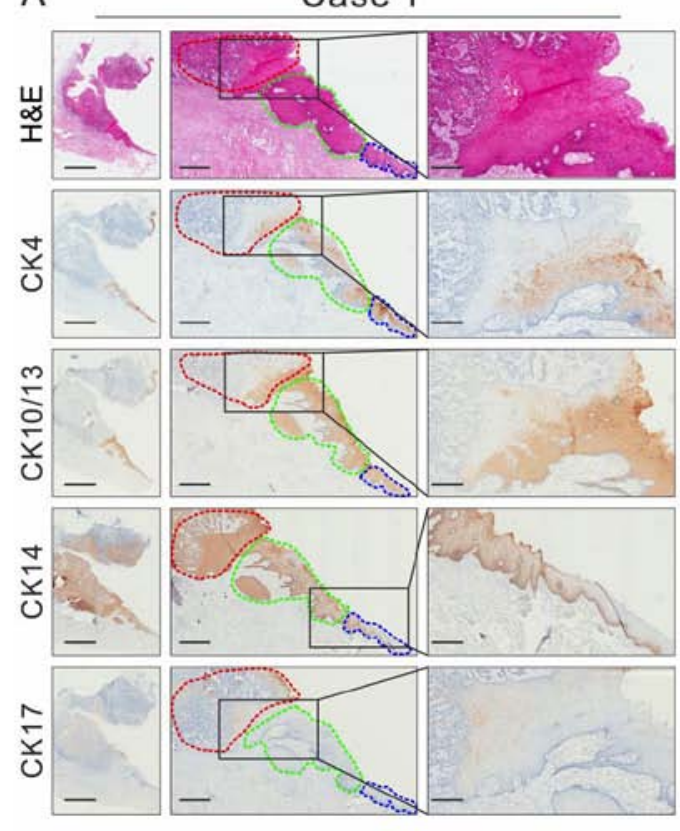

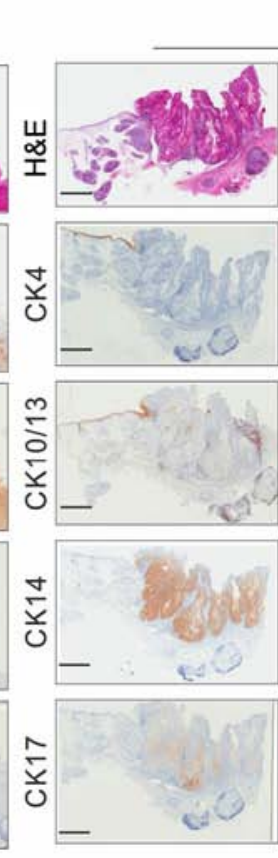

Case 2

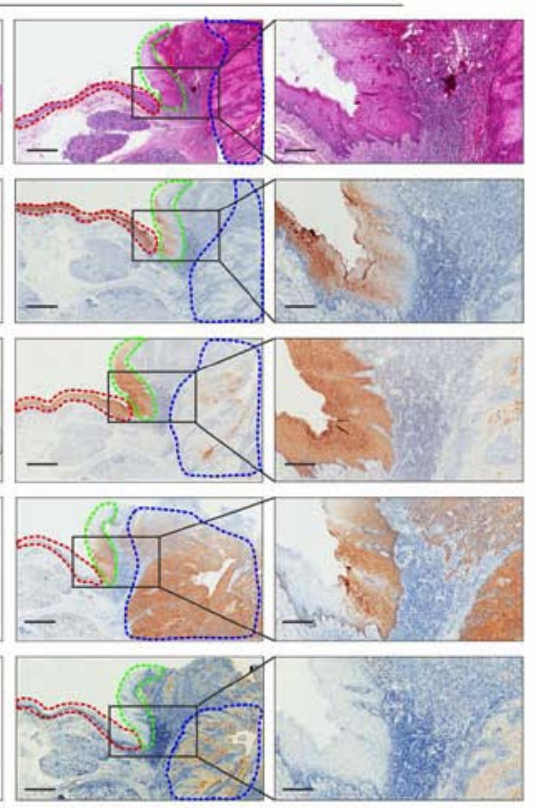

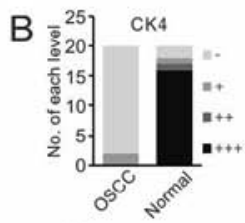
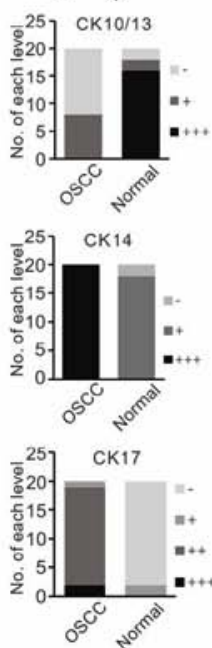

Figure 3. Immunohistochemistry analysis of the identified proteins in clinical tissues. (A) Representative images of CK4, CK10/13, CK14 and CK17 immunostaining of OSCC tissues accompanied by those of adjacent normal/pre-cancerous lesions. The regions were identified by the pathologist: Adjacent normal regions are shown in blue circles, pre-cancerous lesions are shown in green circles and OSCC regions are shown in red circle. Scale bar, $2,000 \mu \mathrm{m}$ (left panels), $1,000 \mu \mathrm{m}$ (middle panels) and $250 \mu \mathrm{m}$ (right panels). (B) Semi-quantitative scores of CK-positive staining in OSCC tissues and adjacent normal tissues of 20 cases. OSCC, oral squamous cell carcinoma; H\&E, hematoxylin and eosin; CK, cytokeratin.

higher in the OSCC tissues than in the normal tissues. In addition, a group of proteins was detected only in OSCC tissues, including myosin and CK18. Certain proteins were detected preferentially in normal tissues, including CK5. Of note, this pattern was in accordance with the previous analysis of OSCC tissues and tumor-adjacent normal tissues.

Expression levels of CK10/13 and 4 or CK14 and 17 are potential molecular markers for the extent of the surgical resection of $O S C C$. Surgical resection beyond contrastenhancing boundaries may represent a promising strategy to improve outcome and quality of life for patients with OSCC. Therefore, a specific molecular marker to guide the determination of surgical margins is urgently required. Using LC/MS proteomics analysis, the present study found significant differences in the expression of CK14, CK17, CK10/13 and CK4 in OSCC tissues, compared with that in normal oral tissues/ para-cancerous tissues. To identify molecular markers that may be used to determine the extent of the surgical resection of OSCC tissue, IHC of CK 10/13, 4, 14 and 17 was performed in 20 FFPE OSCC tissue samples. The expression levels of CK $10 / 13,4,14$ and 17 in two representative cases are shown in Fig. 3A. CK4 was detected at high levels in epithelial cell layers of tumor-adjacent tissues but was not present in OSCC tissues. CK10/13 showed similar distribution patterns in normal and OSCC tissues, but they were also expressed in the basal layers of OSCC tissues. A high level of plasma-positive staining of CK14 and 17 was observed in OSCC tissues, whereas their distribution was confined to the basal layer in the adjacent normal epithelium or in moderate dysplasia. The observation that CK14 and CK17 made it possible to distinguish between
OSCC tissues and adjacent normal tissues/moderate dysplastic tissues indicated a shift in intermediate filament protein expression, which may be a potential diagnostic biomarker for OSCC.

The relative expression levels were evaluated and analyzed (Fig. 3B), and the results showed that negative staining of CK4 and CK10/13 clearly distinguished between cancerous tissue and para-cancerous tissue, with an accuracy of $90 \%(95 \% \mathrm{CI}$, 0.68-0.99) and 75\% (95\% CI, 0.51-0.91), respectively, whereas the positive staining of CK14 and CK17 clearly distinguished between cancerous lesions and para-cancerous lesions with an accuracy of $100 \%$ (95\% CI, 83-100\%) and 90\% (95\% CI, 0.68-0.99), respectively. These results suggested that CK4 and CK10/13 or CK14 and CK17 may be useful molecular boundary markers for guidance in the surgical resection of OSCC.

CK14 is a superior predictor of OSCC lymph node micrometastases compared with Pan-CK. A previous study indicated that CK14 was upregulated in oral cancer tissues (15). Therefore, the present study further evaluated the potential use of CK14 for the detection of lymph node metastasis. As shown in Fig. 4A, CK14 was specifically positive for micrometastasis in all five cases. Pan-CK, an important predictor of lymph node metastasis in OSCC, was used as a control. An expanded set of 20 samples was further examined. The IHC of CK14 was specifically positive for micrometastasis in all cases and was more specific than Pan-CK (Fig. 4B). In addition, 10 samples diagnosed as non-metastatic lymph nodes were examined, and it was found that seven cases were CK14-positive. One of the seven CK14-positive cases showed recurrence 1 year later (Fig. 4C). Collectively, the results from the lymph node 

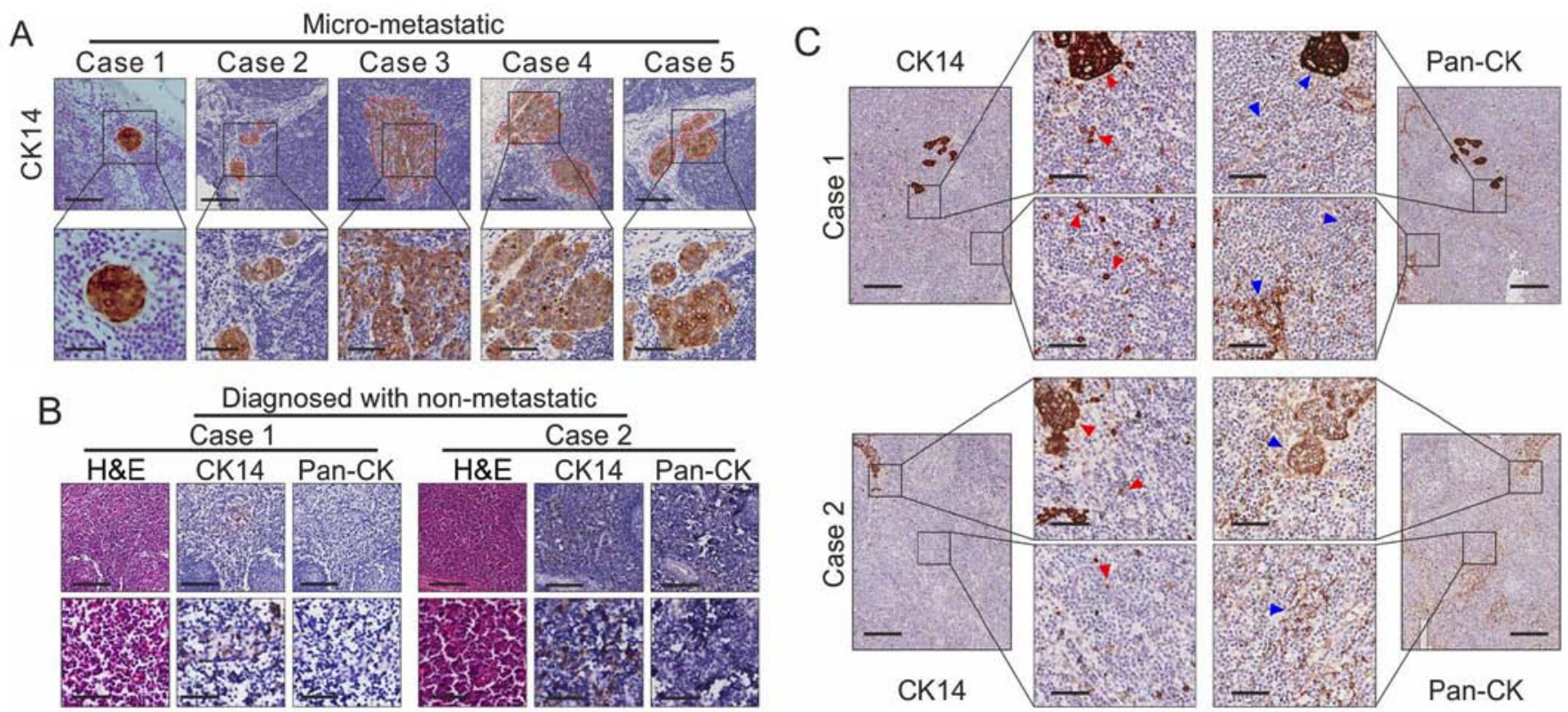

Figure 4. CK14 immunostaining in lymph nodes of clinical patients. (A) Representative images of CK14 immunostaining in lymph nodes with micrometastases from patients with OSCC. Scale bar, $500 \mu \mathrm{m}$ (upper panels) and $100 \mu \mathrm{m}$ (lower panels). (B) Representative images of H\&E staining and CK14 and Pan-CK immunostaining in lymph nodes diagnosed as non-metastatic from patients with OSCC. Scale bar, $100 \mu \mathrm{m}$ (left panels), $500 \mu \mathrm{m}$ (two middle panels) and $100 \mu \mathrm{m}$ (right panels). (C) Representative images of CK14 and Pan-CK immunostaining in lymph node tissues from patients with OSCC. The arrows indicated the staining of CK14 (red) and Pan-CK (blue). Scale bar, $500 \mu \mathrm{m}$ (upper panels) and $200 \mu \mathrm{m}$ (lower panels). OSCC, oral squamous cell carcinoma; $\mathrm{H} \& \mathrm{E}$, hematoxylin and eosin; CK, cytokeratin.
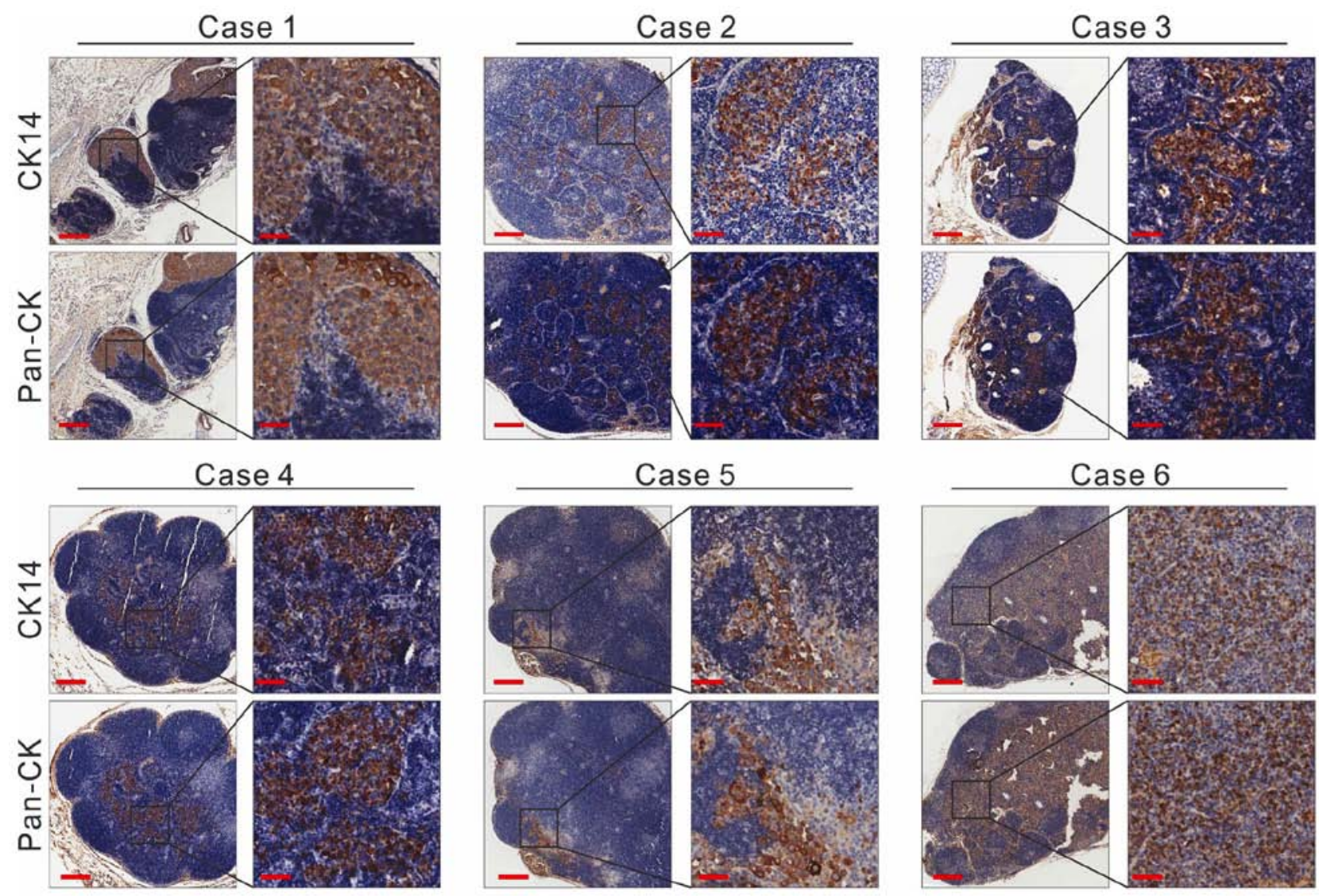

Figure 5. CK14 immunostaining in lymph nodes from a mouse model. Scale bar, $200 \mu \mathrm{m}$ (left panels) and $50 \mu \mathrm{m}$ (right panels). CK, cytokeratin.

metastasis mouse model data were consistent with the clinical results (Fig. 5). These results indicated the potential value of
CK14 as a biomarker for the detection of lymph node micrometastasis in OSCC. 


\section{Discussion}

The discrimination of OSCC tissues from para-cancerous tissues in $\mathrm{H} \& \mathrm{E}$ sections can be difficult due to the subjectivity of the microscopic analyses, particularly when the histologic structure is irregularly distributed. The majority of surgeons use a $0.5-1 \mathrm{~cm}$ empirical boundary to expand the excision of OSCC $(16,17)$. However, in maxillofacial surgery, this can lead to the irreversible destruction of the facial appearance, impacting the quality of life of the patients. Therefore, identifying specific molecular markers to guide the determination of surgical margins is urgently required $(18,19)$.

A wealth of information has been provided by intensive research efforts in cancer biomarker identification based on the LCM-FFPE-proteomic analysis. In previous studies that focused on the utility of proteomic analysis based on FFPE tissue, it was suggested that this platform was an effective approach for biomarker identification in cancer. In the present study, the expression profiles of a large number of proteins were determined, revealing significant shifts in expression between normal mucosal epithelial tissues and pre-cancerous and cancer tissues. A total of 13 proteins were identified with altered expression levels based on LCM-FFPE-proteomic and LC/MS analyses. Of these, the expression patterns of CK14, 17,4 and 10/13 were found to have unique patterns that may serve as biomarkers for the histologic evaluation of oral mucosa, particularly when histologic structures are unclear. The results of the IHC and H\&E staining were consistent, and CK14, 17, 4 and 10/13 staining made it possible to distinguish between early phenotype changes in tumor cells and adjacent cells, which are difficult to detect by conventional H\&E staining.

CKs are often used as a source for the diagnosis/differential diagnosis of epithelial-derived tumors (20). Several studies have reported that these expression patterns correlate with the presence of OSCC and oral epithelial dysplasia. Previous reports have suggested that the downregulated expression of CK4 and CK13 in OSCC may cause morphological alterations in oral carcinogenesis and are relevant biomarkers of OSCC $(8,21)$. The expression patterns of CK13, 4, 14, and 17 during the development of OSCC from oral precancerous lesions have also been investigated (22-24). The results of the present study were consistent with reports. In previous studies, CK14 was often expressed in myoepithelial cells and other epithelial cells, whereas CK10/13 was mainly expressed in the stratified squamous epithelium $(25,26)$. The results of the statistical analysis in the present study confirmed the results of these previous studies, demonstrating that CK14, 17, 4 and 10/13 staining can distinguish between early phenotypic changes in tumor cells and adjacent cells, which are difficult to detect by conventional H\&E staining.

$\mathrm{H} \& \mathrm{E}$ staining is the current standard for the diagnosis of OSCC. However, the discrimination of micrometastasis in lymph nodes based on H\&E-stained sections of OSCC is difficult. Therefore, the identification of relatively objective biomarkers that can be used to identify micrometastasis in lymph nodes is likely to benefit patients with OSCC. The expression of CK14 is altered in several diseases, including human papillomavirus infections, lichen planus, psoriasis, inflammation, and metaplasia (27-30). The data from the clinical samples and a mouse model in the present study showed for the first time, to the best of our knowledge, that CK14 has potential value as a biomarker for the detection of lymph node micrometastasis in OSCC. Additionally, signals for CK14 were found in a high proportion of lymph nodes diagnosed as non-metastatic from patients with OSCC who developed micro-metastases. One of the CK14-positive cases showed recurrence 1 year later, which may partly explain the significant correlations between locoregional recurrence and the number of resected lymph nodes and lymph node ratio of patients with oral cavity cancer (31). The number of resected lymph nodes is a prognosticator of multiple types of cancer, including vulvar cancer (32), gastric cancer (33) and non-small cell lung cancer (34). The data in the present study are in agreement with those described previously. Larger investigations with larger clinical cohorts are required prior to CK14 being used as a clinical micrometastasis biomarker in the future.

In conclusion, the data obtained in the present study indicated that the expression levels of CK10/13/4 or CK14/17 may be considered molecular markers to guide the extent of surgical resection of OSCC. The major finding of the present study was that CK14 is a superior predictor of lymph node micro-metastases in OSCC, performing better than Pan-CK. Taken together, the data obtained indicated that the platform based on LCM-FFPE-proteomic analysis is an effective approach for biomarker identification in OSCC.

\section{Acknowledgements}

The authors would like to thank Dr Vyomesh Patel (National Institutes of Health, National Institute of Dental and Craniofacial Research, Bethesda, MD, USA), Dr Li-hong Yao, Dr Ming-zhong Yang and Dr Gao Wu (University of Science and Technology of China, Anhui, China) for their technical assistance and advice.

\section{Funding}

The present study was supported by the National Natural Science Foundation of China (grant nos. 81621062, 81321002, 81621062,81672675 and 81472533), the 111 Project of MOE China (grant no. B14038), the National Natural Science Foundation of China-Canadian Institutes of Health Research (grant no. 81010066) and the Open Foundation of State Key Laboratory of Oral Diseases, Sichuan University (grant nos. SKLOD201701 and SKLOD201714).

\section{Availability of data and materials}

The datasets used during the present study are available from the corresponding author upon reasonable request.

\section{Authors' contributions}

$\mathrm{RW}, \mathrm{YZ}$ and NG performed the LCM and the proteomic analysis; RW, DZ, NJ, YZ, MZ, YY and JL performed the IHC experiments. XL, QC, NG, XZ, YY and JL conceived, designed, analyzed and interpreted the data; LZ, YC and NG performed the pathological diagnosis of the patients. $\mathrm{XZ}$ and $M Z$ were responsible for the tissue collection and management. YZ, LZ and QC wrote and revised the manuscript. All authors 
read and approved the manuscript and agree to be accountable for all aspects of the research in ensuring that the accuracy or integrity of any part of the work are appropriately investigated and resolved.

\section{Ethics approval and consent to participate}

The use of animals in the experiments was approved by the State Key Laboratory of Oral Diseases Research Ethics Committee. The Institutional Review Board of the West China College of Stomatology, Sichuan University approved the protocol.

\section{Patient consent for publication}

Not applicable.

\section{Competing interests}

The authors declare that they have no competing interests.

\section{References}

1. Warnakulasuriya S: Global epidemiology of oral and oropharyngeal cancer. Oral Oncol 45: 309-316, 2009.

2. Chen W, Zheng R, Baade PD, Zhang S, Zeng H, Bray F, Jemal A, $\mathrm{Yu}$ XQ and He J: Cancer statistics in China, 2015. CA Cancer J Clin 66: 115-132, 2016.

3. Panzarella V, Pizzo G, Calvino F, Compilato D, Colella G and Campisi G: Diagnostic delay in oral squamous cell carcinoma: The role of cognitive and psychological variables. Int J Oral Sci 6: 39-45, 2014

4. Perez-Ordoñez B, Beauchemin M and Jordan RC: Molecular biology of squamous cell carcinoma of the head and neck. J Clin Pathol 59: 445-453, 2006

5. Messadi DV: Diagnostic aids for detection of oral precancerous conditions. Int J Oral Sci 5: 59-65, 2013.

6. Warnakulasuriya S,Johnson NW and van derWaal I: Nomenclature and classification of potentially malignant disorders of the oral mucosa. J Oral Pathol Med 36: 575-580, 2007.

7. Wulfkuhle JD, Liotta LA and Petricoin EF: Proteomic applications for the early detection of cancer. Nat Rev Cancer 3: 267-275, 2003

8. Schaaij-Visser TB, Bremmer JF, Braakhuis BJ, Heck AJ, Slijper M, van der Waal I and Brakenhoff RH: Evaluation of cornulin, keratin 4, keratin 13 expression and grade of dysplasia for predicting malignant progression of oral leukoplakia. Oral Oncol 46: 123-127, 2010.

9. Hood BL, Conrads TP and Veenstra TD: Mass spectrometric analysis of formalin-fixed paraffin-embedded tissue: Unlocking the proteome within. Proteomics 6: 4106-4114, 2006.

10. Sprung RW Jr, Brock JW, Tanksley JP, Li M, Washington MK, Slebos RJ and Liebler DC: Equivalence of protein inventories obtained from formalin-fixed paraffin-embedded and frozen tissue in multidimensional liquid chromatography-tandem mass spectrometry shotgun proteomic analysis. Mol Cell Proteomics 8: 1988-1998, 2009.

11. Yuan Y, Hou X, Feng H, Liu R, Xu H, Gong W, Deng J, Sun C, Gao Y, Peng J, et al: Proteomic identification of cyclophilin A as a potential biomarker and therapeutic target in oral submucous fibrosis. Oncotarget 7: 60348-60365, 2016.

12. Espina V, Wulfkuhle JD, Calvert VS, VanMeter A, Zhou W, Coukos G, Geho DH, Petricoin EF III and Liotta LA: Lasercapture microdissection. Nat Protoc 1: 586-603, 2006.

13. Merkley MA, Weinberger PM, Jackson LL, Podolsky RH, Lee JR and Dynan WS: 2D-DIGE proteomic characterization of head and neck squamous cell carcinoma. Otolaryngol Head Neck Surg 141: 626-632, 2009.

14. Pawar H, Maharudraiah J, Kashyap MK, Sharma J, Srikanth SM, Choudhary R, Chavan S, Sathe G, Manju HC, Kumar KV, et al: Downregulation of cornulin in esophageal squamous cell carcinoma. Acta Histochem 115: 89-99, 2013.
15. Shores CG, Yin X, Funkhouser W and Yarbrough W: Clinical evaluation of a new molecular method for detection of micrometastases in head and neck squamous cell carcinoma. Arch Otolaryngol Head Neck Surg 130: 937-942, 2004.

16. Loree TR and Strong EW: Significance of positive margins in oral cavity squamous carcinoma. Am J Surg 160: 410-414, 1990.

17. Cheng A, Cox D and Schmidt BL: Oral squamous cell carcinoma margin discrepancy after resection and pathologic processing. J Oral Maxillofac Surg 66: 523-529, 2008.

18. Hosseinpour S, Mashhadiabbas F and Ahsaie MG: Diagnostic biomarkers in oral verrucous carcinoma: A systematic review. Pathol Oncol Res 23: 19-32, 2017.

19. Tirelli G, Zacchigna S, Biasotto M and Piovesana M: Open questions and novel concepts in oral cancer surgery. Eur Arch Otorhinolaryngol 273: 1975-1985, 2016.

20. Khanom R, Sakamoto K, Pal SK, Shimada Y, Morita K, Omura K, Miki Y and Yamaguchi A: Expression of basal cell keratin 15 and keratin 19 in oral squamous neoplasms represents diverse pathophysiologies. Histol Histopathol 27: 949-959, 2012.

21. Sakamoto K, Aragaki T, Morita K, Kawachi H, Kayamori K, Nakanishi S, Omura K, Miki Y, Okada N, Katsube K, et al: Down-regulation of keratin 4 and keratin 13 expression in oral squamous cell carcinoma and epithelial dysplasia: A clue for histopathogenesis. Histopathology 58: 531-542, 2011

22. Ohkura S, Kondoh N, Hada A, Arai M, Yamazaki Y, Sindoh M, Takahashi M, Matsumoto I and Yamamoto M: Differential expression of the keratin-4, $-13,-14,-17$ and transglutaminase 3 genes during the development of oral squamous cell carcinoma from leukoplakia. Oral Oncol 41: 607-613, 2005.

23. Nobusawa A, Sano T, Negishi A, Yokoo S and Oyama T: Immunohistochemical staining patterns of cytokeratins 13 , 14 , and 17 in oral epithelial dysplasia including orthokeratotic dysplasia. Pathol Int 64: 20-27, 2014.

24. Valach J, Foltan R, Vlk M, Szabo P and Smetana K Jr: Phenotypic characterization of oral mucosa: What is normal? J Oral Pathol Med 46: 834-839, 2017.

25. Hansson A, Bloor BK, Haig Y, Morgan PR, Ekstrand J and Grafström RC: Expression of keratins in normal, immortalized and malignant oral epithelia in organotypic culture. Oral Oncol 37: 419-430, 2001.

26. Ming XY, Fu L, Zhang LY, Qin YR, Cao TT, Chan KW, Ma S, Xie D and Guan XY: Integrin $\alpha 7$ is a functional cancer stem cell surface marker in oesophageal squamous cell carcinoma. Nat Commun 7: 13568, 2016.

27. Jacques CM, Pereira AL, Maia V, Cuzzi T and Ramos-e-Silva M: Expression of cytokeratins 10,13,14 and 19 in oral lichen planus. J Oral Sci 51: 355-365, 2009.

28. Fay J, Kelehan P, Lambkin $\mathrm{H}$ and Schwartz S: Increased expression of cellular RNA-binding proteins in HPV-induced neoplasia and cervical cancer. J Med Virol 81: 897-907, 2009

29. Bech R, Otkjaer K, Birkelund S, Vorup-Jensen T, Agger R, Johansen C, Iversen L, Kragballe K and Rømer J: Interleukin 20 protein locates to distinct mononuclear cells in psoriatic skin. Exp Dermatol 23: 349-352, 2014.

30. Linskey KR, Gimbel DC, Zukerberg LR, Duncan LM, Sadow PM and Nazarian RM: BerEp4, cytokeratin 14, and cytokeratin 17 immunohistochemical staining aid in differentiation of basaloid squamous cell carcinoma from basal cell carcinoma with squamous metaplasia. Arch Pathol Lab Med 137: 1591-1598, 2013.

31. Safi AF, Grandoch A, Nickenig HJ, Zöller JE and Kreppel M: The importance of lymph node ratio for locoregional recurrence of squamous cell carcinoma of the tongue. J Craniomaxillofac Surg 45: 1058-1061, 2017.

32. Polterauer S, Schwameis R, Grimm C, Macuks R, Iacoponi S, Zalewski K and Zapardiel I; VULCAN Study Collaborative Group: Prognostic value of lymph node ratio and number of positive inguinal nodes in patients with vulvar cancer. Gynecol Oncol 147: 92-97, 2017.

33. Gholami S, Janson L, Worhunsky DJ, Tran TB, Squires MH III, Jin LX, Spolverato G, Votanopoulos KI, Schmidt C, Weber SM, et al: Number of lymph nodes removed and survival after gastric cancer resection: An analysis from the US gastric cancer collaborative. J Am Coll Surg 221: 291-299, 2015.

34. Liang W, He J, Shen Y, Shen J, He Q, Zhang J, Jiang G, Wang Q, Liu L, Gao S, et al: Impact of examined lymph node count on precise staging and long-term survival of resected non-small-cell lung cancer: A population study of the us seer database and a chinese multi-institutional registry. J Clin Oncol 35: 1162-1170, 2017. 